1

\title{
Estimates of reduced vaccine effectiveness against hospitalization, infection, transmission and symptomatic disease of a new SARS-CoV-2 variant, Omicron (B.1.1.529), using neutralizing antibody titers
}

\author{
Billy J. Gardner, A. Marm Kilpatrick*
}

Department of Ecology and Evolutionary Biology, University of California, Santa Cruz, California, USA

*akilpatr@ucsc.edu

\begin{abstract}
The emergence of the Omicron variant (B.1.1.529) of SARS-CoV-2 has raised concerns about how mutations in the spike protein might influence immune escape and vaccine protection against infection and disease, COVID-19. Initial estimates of immune escape measure neutralizing antibody titers, which have been shown to be a correlate of protection for COVID-19, but vary among studies. However, no studies have examined variation in vaccine effectiveness (VE) using estimated reductions in neutralizing antibody titers across virus variants. We quantified consistency in relative neutralizing antibody titers across studies. We then examined relationships between variant-specific reductions in neutralizing antibodies and protection against documented infection, symptomatic disease, and hospitalizations across variants and vaccines. We found considerable variation in variant-specific neutralizing antibody titers between studies, but within-study comparisons across variants were far more robust. There was insufficient data to estimate VE for a single vaccine across variants, especially for higher levels of immune evasion (>7-fold reductions in neutralizing antibody titers) observed with the Omicron variant (40-fold). Instead, we leveraged variation among both vaccines and virus variants to estimate VE - neutralizing antibody titer relationships across a 30 to 100 -fold range of neutralizing antibody titers reduction. Omicron increased the risk of hospitalization four to five-fold and increased the risk of symptomatic disease seven to ten-fold for mRNA vaccinees, with similar relative effects for recently vaccinated, or individuals with waned antibody titers. Third doses restored titers and protection to levels similar to waned immunity against Delta. Overall, these analyses indicate that vaccine effectiveness against severe disease is significantly diminished for waned individuals, and protection against infection, symptomatic disease and transmission is nearly eliminated. However, third doses significantly ameliorate these reductions but only restore protection to levels equivalent to waned protection against the Delta variant. The invasion of Omicron is likely to result in widespread infection, and substantial hospitalizations unless widespread boosting of immunity occurs.
\end{abstract}

Funding California Department of Health, National Science Foundation 


\section{Introduction}

The evolution of SARS-CoV-2 in populations with high immunity from infection or vaccination is likely to lead to new variants with substantial immune escape $(1,2)$, including the Omicron variant (B.1.1.529) in November 2021. Determining the extent of immune evasion on the effectiveness of vaccines is critical both for assessing the need for new and variant-specific vaccines and additional non-pharmaceutical interventions to limit spread(3). Traditional vaccine effectiveness studies can be costly and can only be performed when there is significant transmission of a new variant in a partly vaccinated population. Delays in implementing interventions until these studies can be performed can result in rapid growth of a new variant, stress on health-care systems and preventable disease and death (3).

A much quicker way to estimate VE against a new variant is to use a surrogate of protection, such as neutralizing antibody titers, which can often be measured much more quickly than performing a traditional VE study. Variation in neutralizing antibody titers across vaccines are strongly correlated with protection against symptomatic disease, all infections, and transmission (4-7). Here, we extend this approach and develop relationships between VE against documented infection, symptomatic disease, and hospitalization and neutralizing antibody titers. We use these relationships to estimate the impact of immune evasion, specifically, reductions in neutralizing antibody titers on VE, including for the Omicron variant.

\section{Methods}

Reductions in neutralizing antibody titers by variant

We collected data from a recent review (8) on neutralizing antibody titers for four SARSCoV-2 variants, Alpha, Beta, Gamma, and Delta (Table S1), and for each vaccine, normalized by convalescent sera (4). We excluded studies that did not include values for at least 3 variants because between-study variation was very high (see below). We divided each estimate by the neutralizing antibody titer for wild-type (WT) SARS-CoV-2 (Wuhan-Hu-1, US-WA1/2020, B, B.1) from each study, to produce a ratio representing the reduction (expressed as an $\mathrm{x}$-fold difference) between a variant and WT virus. We estimated the mean differences among variants using a linear model with variant as a fixed effect (a mixed model with study as a random effect had weaker support; AIC: 0.7 ). We used these variant-specific reduction in neutralizing antibody levels (relative to WT) and the neutralizing antibody levels for each vaccine relative to convalescent sera (4) to estimate a vaccine-variant specific relative neutralizing antibody titer ratio, NATR.

\section{VE against documented infection, symptomatic disease, and hospitalization}

We collected data from the literature (including a systematic living review: (9)) on VE for COVID-19 and categorized each study by variant type (Alpha, Beta, Gamma, Delta) and endpoint (hospitalization, symptomatic disease, and documented infection). We excluded estimates where a dominant variant for the study period was not reported, or where two variants were circulating and no variant-specific estimate was provided. 
medRxiv preprint doi: https://doi.org/10.1101/2021.12.10.21267594; this version posted December 12, 2021. The copyright holder for this preprint (which was not certified by peer review) is the author/funder, who has granted medRxiv a license to display the preprint in perpetuity.

It is made available under a CC-BY-NC-ND 4.0 International license .

87

\section{Relationships between VE and neutralizing antibody titers by vaccine and variant}

We used generalized linear models with a binomial distribution with a logit link to model the relationship between variant-specific reductions in neutralizing antibody titers and VE for each endpoint across all vaccines and variants for each endpoint. We used these relationships to estimate VE for three endpoints, documented infection, symptomatic disease, and hospitalization, for the Omicron variant, using a $\sim 40$-fold reduction in neutralizing antibody titers relative to WT (10-12). We also estimated VE for all infections and transmission given infection using previously identified relationships between VE and neutralizing antibody titers (5).

All code and data to replicate the results can be found at https://github.com/marmkilpatrick/New-Variant-VE.

\section{Results}

There was enormous variation in the ratios of neutralizing antibody titers to WT (5-10fold), but variation among variants in normalized titers was much smaller (Fig 1). Neutralizing antibody titers relative to WT were1.86-fold (95\% Cl: 1.3-2.4) lower for Alpha, 6.7-fold (95\% Cl: 5.0-9.1) lower for Beta, 2.3-fold (95\% Cl: 5.0-9.1) for Gamma, and 2.7-fold (95\% Cl: 2.7-5.1) lower for Delta (Fig 1).

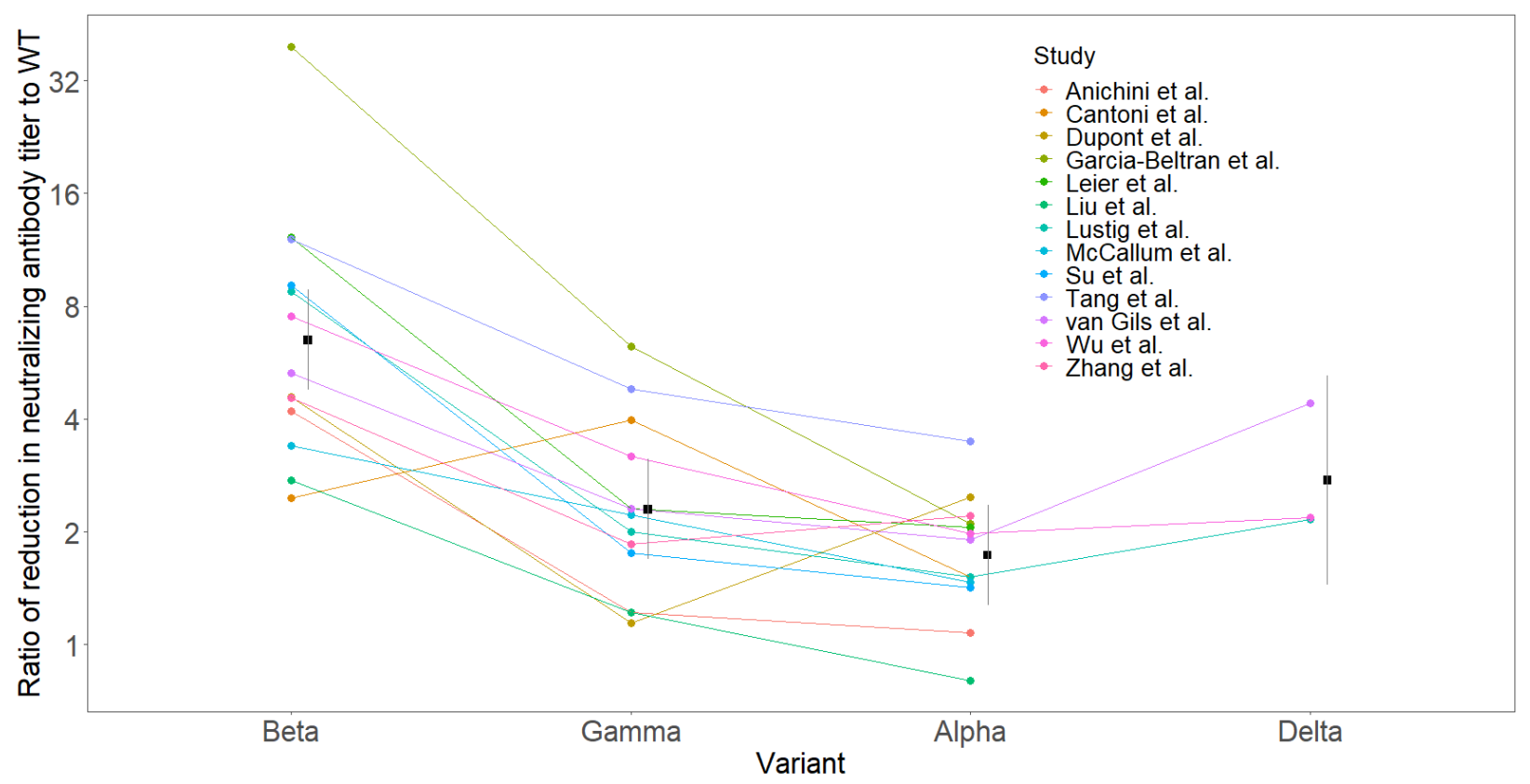

Fig 1. Reduction in neutralizing antibody titers plotted against SARS-CoV-2 variant. Each point and color show ratio from an individual study, and points from the same study are connected by lines. Black squares and error bars show the 
medRxiv preprint doi: https://doi.org/10.1101/2021.12.10.21267594; this version posted December 12, 2021. The copyright holder for this preprint (which was not certified by peer review) is the author/funder, who has granted medRxiv a license to display the preprint in perpetuity.

It is made available under a CC-BY-NC-ND 4.0 International license .

mean and $95 \%$ confidence intervals for each variant. Means and $95 \%$ confidence intervals are $1.73(95 \% \mathrm{Cl} 1.27-2.36)$ for Alpha, 6.50 (95\% Cl: 4.77-8.85) for Beta, 2.74 (95\% Cl: 1.44-5.22) for Delta, and 2.30 (1.69-3.13) for Gamma.

There were VE estimates for five vaccines against 2 to 4 variants for hospitalization (Fig 2), symptomatic disease (Fig 3), and documented infection (Fig 4). In some cases there was evidence for reductions in VE with decreasing neutralizing antibody titers, but for hospitalization and symptomatic disease endpoints the VE estimates for the variant with the largest reduction in neutralizing antibody titers (Beta) were highly uncertain, resulting in a vary narrow range of neutralizing antibody titers, and insufficient information to estimate the VE for Omicron based solely on comparisons within a vaccine across variants.

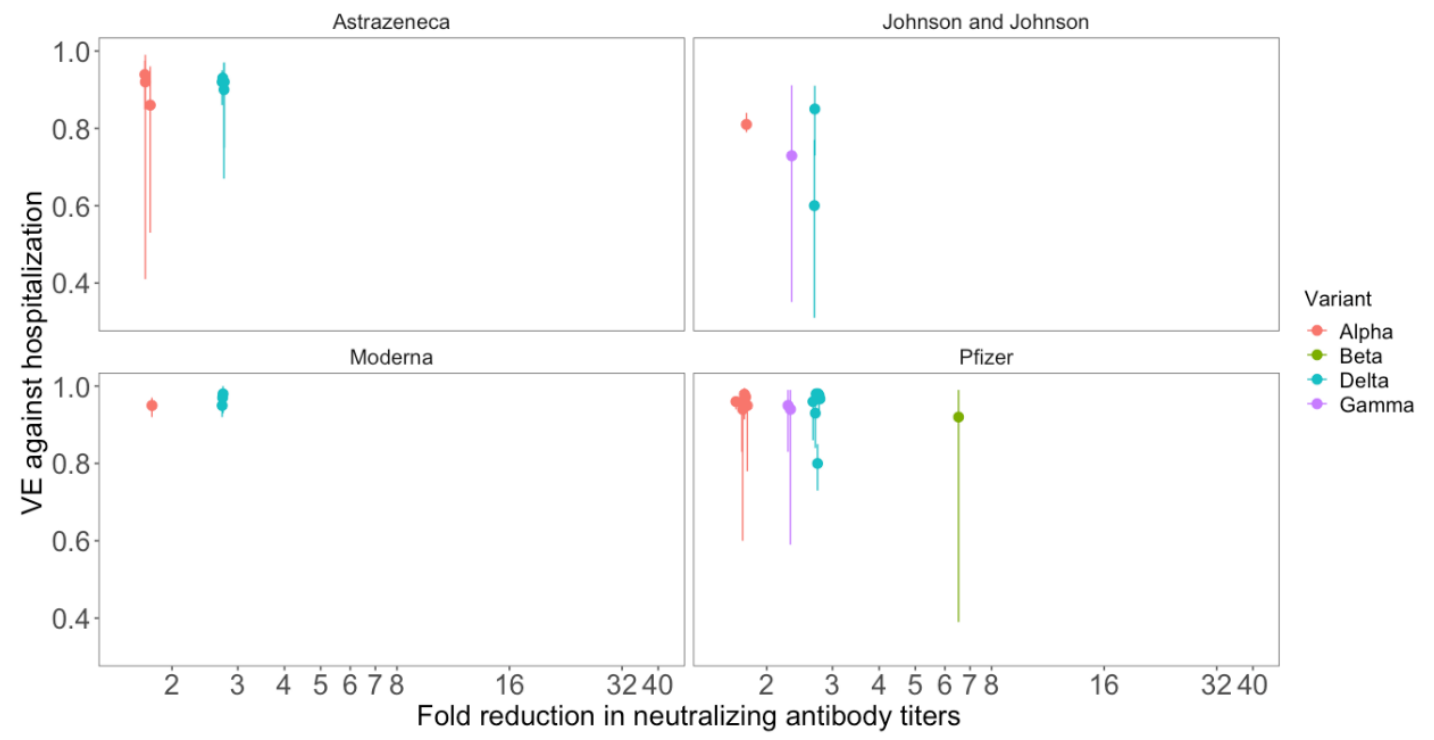

Fig 2. VE against hospitalization plotted against variant-specific reductions in neutralizing antibody titers by vaccine. Each point (and $95 \% \mathrm{Cl}$ ) represents a single estimate of VE for a single vaccine \& virus variant from observational studies. Points have been jittered along the $x$-axis to facilitate presentation. 


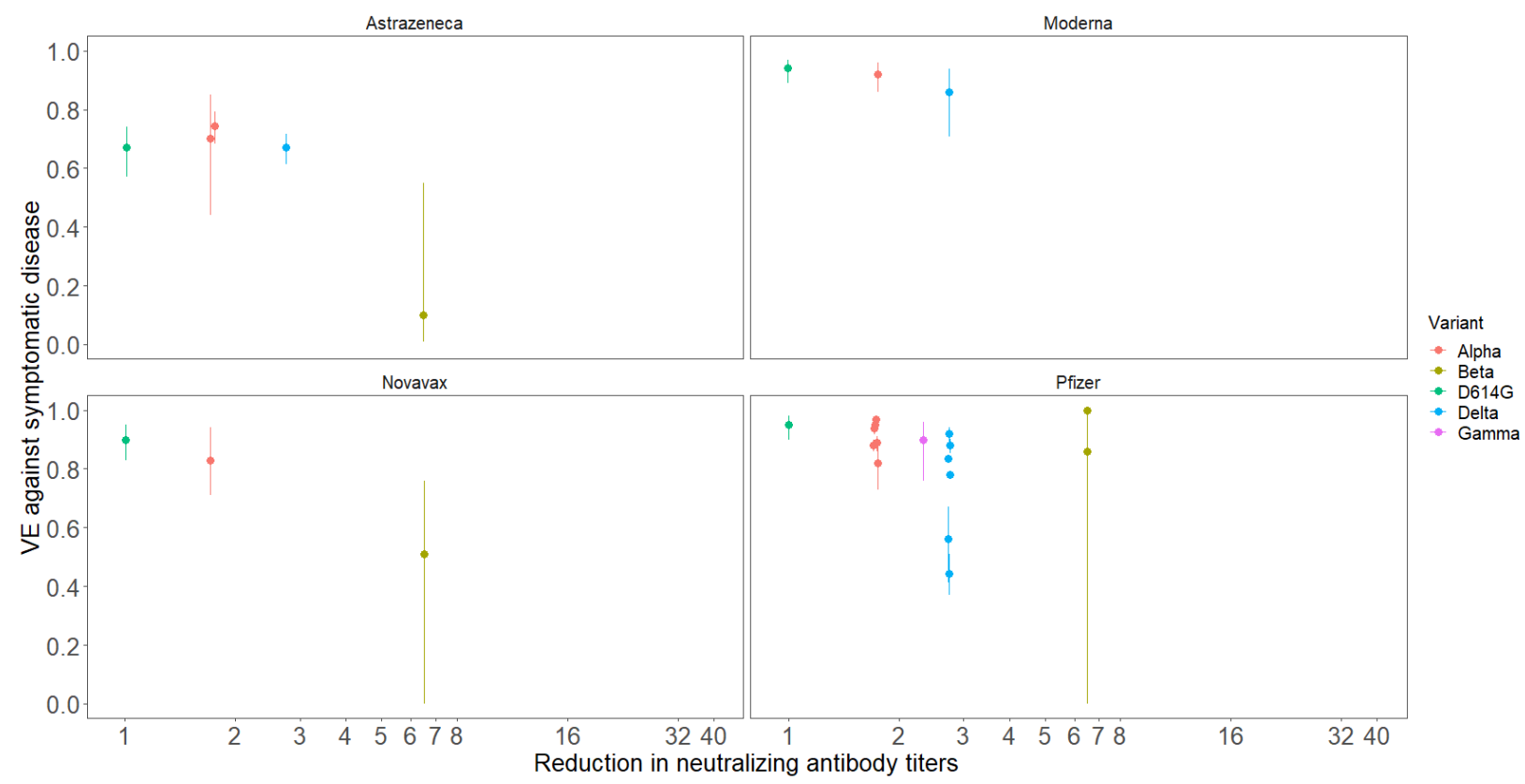

Fig 3. VE against symptomatic disease plotted against variant-specific reductions in neutralizing antibody titers by vaccine. Each point (and $95 \% \mathrm{Cl}$ ) represents a single estimate of VE for a single vaccine \& virus variant from observational studies. Points have been jittered along the $x$-axis to facilitate presentation.

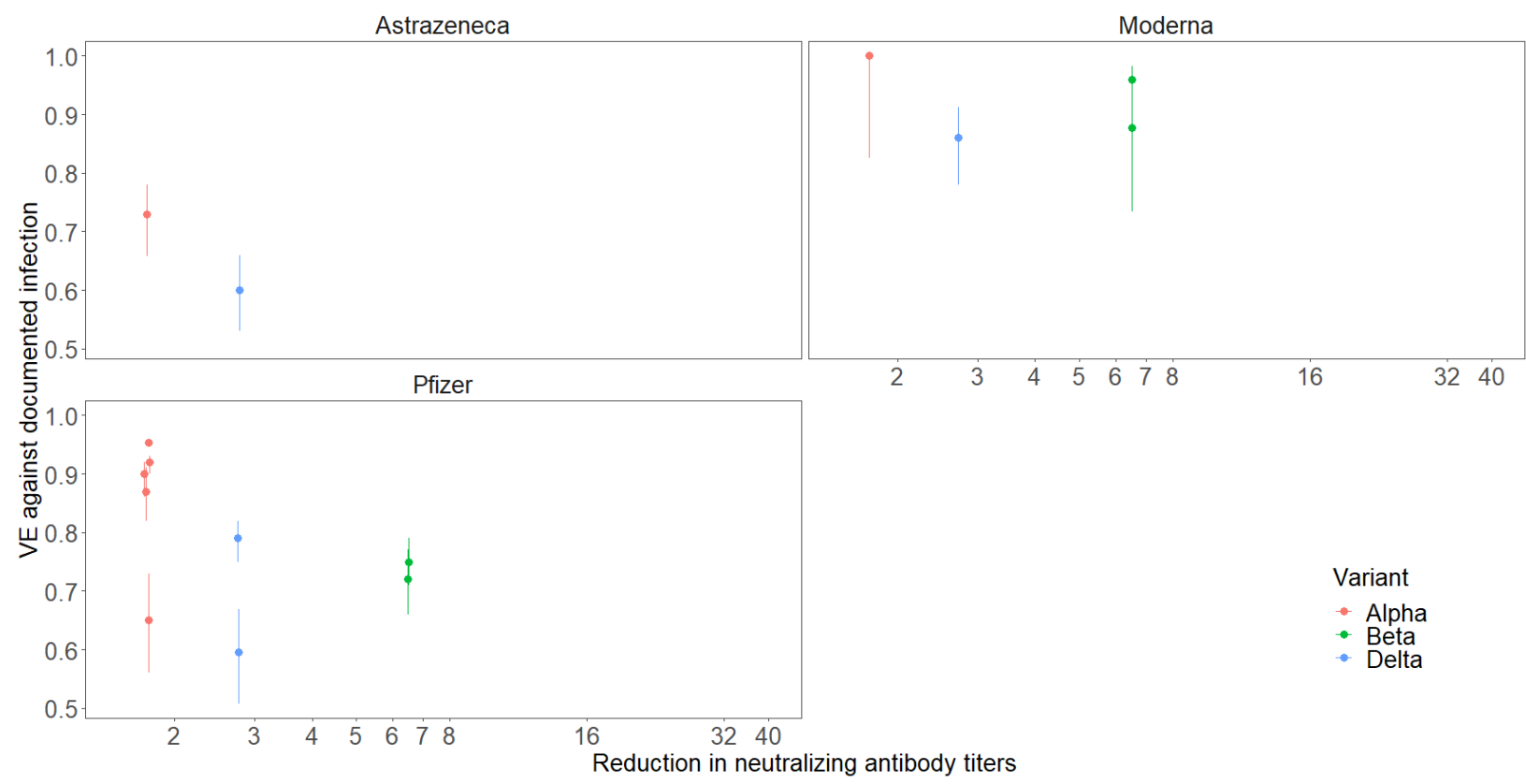

142 Fig 4. VE against documented infection plotted against variant-specific

143 reductions in neutralizing antibody titers by vaccine. Each point (and $95 \% \mathrm{Cl}$ ) represents a single estimate of VE for a single vaccine \& virus variant from 
medRxiv preprint doi: https://doi.org/10.1101/2021.12.10.21267594; this version posted December 12, 2021. The copyright holder for this preprint (which was not certified by peer review) is the author/funder, who has granted medRxiv a license to display the preprint in perpetuity.

It is made available under a CC-BY-NC-ND 4.0 International license .

\section{observational studies. Points have been jittered along the $\mathrm{x}$-axis to facilitate} presentation.

When we analyzed variation in VE with neutralizing antibody titer ratios across both variants and vaccines there were strong relationships across a 30 to 110-fold range of neutralizing antibody titers for the three endpoints (Fig 5A-C). Estimated VE against hospitalization for Omicron was substantially lower than Delta, with relative risk (1-VE) increasing four to five-fold for both Pfizer and Moderna across all vaccination states (Table S3; Fig 6). For example, VE was estimated to be 96.3\% (95\% Cl: 96.1-96.6) against the Delta variant for recently vaccinated Pfizer recipients but $84.9 \%(95 \% \mathrm{Cl}$ : 83.0-86.6) against the Omicron variant (Table S3; Fig. 6). Waned VE was 88.8\% (95\% Cl: 87.7-89.8) for Delta but dropped to an extrapolated 63.1\% (95\% Cl: 56.9-68.9) w/ Omicron. Fortunately, a third Pfizer dose reversed much of the loss in protection and increased VE to $91.7 \%$ (95\% Cl: 91.0-92.2) against Omicron, which was slightly higher than waned Pfizer VE against Delta (Fig 6). Relative patterns were similar for Moderna vaccinees, except VE was a bit higher than Pfizer for recently vaccinated and waned, but boosting was less effective, resulting in VE being similar for both Omicron and Delta for Pfizer and Moderna vaccinees (Fig 6).

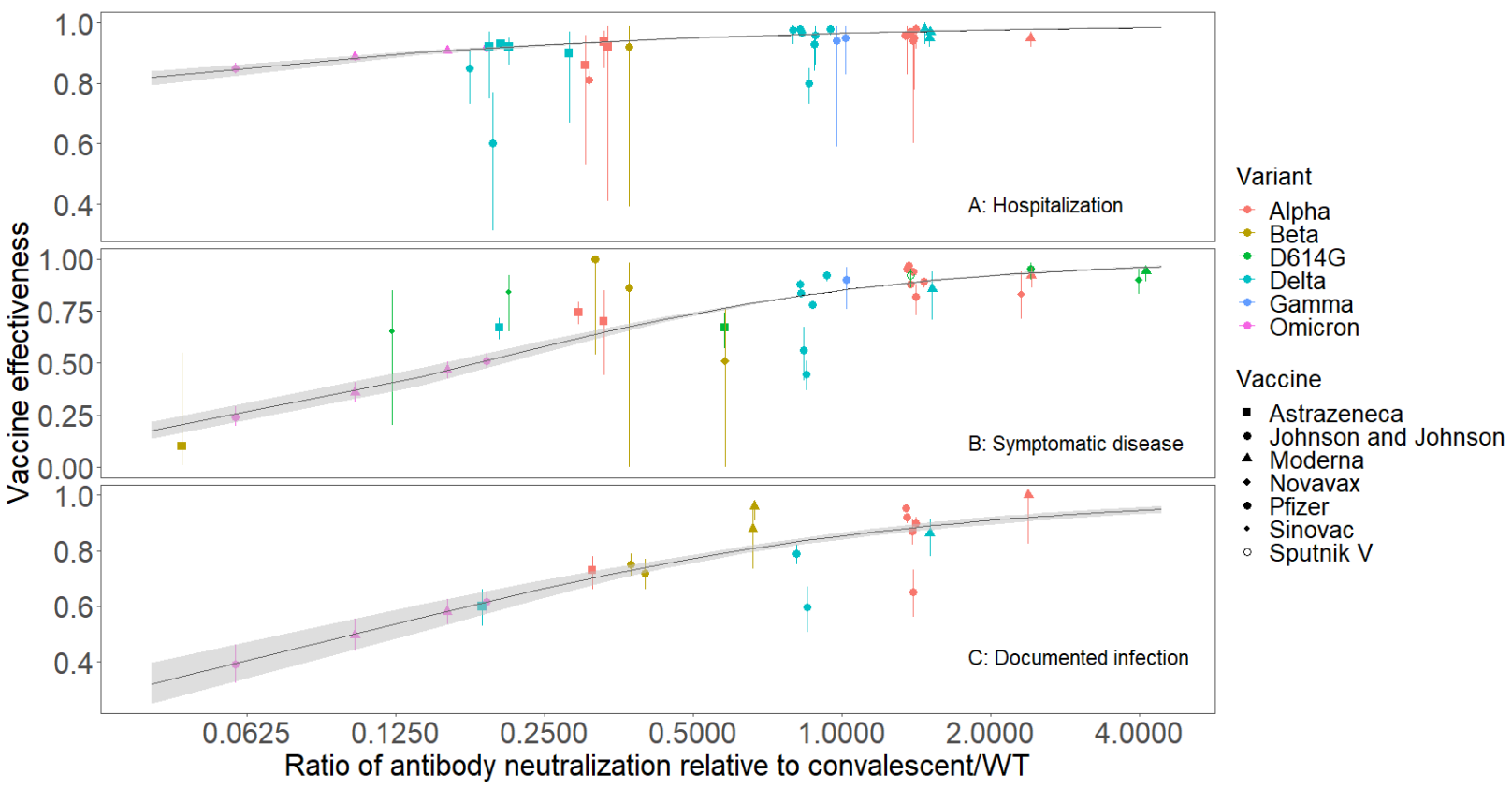

Fig 5. VE against (A) hospitalization (B) symptomatic disease, and (C) documented infection, plotted against variant- and virus-specific neutralizing antibody titer ratios by vaccine. Each point (and $95 \% \mathrm{Cl}$ ) represents a single estimate of VE for a single vaccine \& virus variant from observational studies. Points have been jittered along the $x$-axis to facilitate presentation. Lines (and 95\% $\mathrm{Cl}$ ribbons) show fitted logistic regression models (A: logit(VE_hosp.) = $3.35+0.397^{\star} \log _{2}$ (Neut Ab); $P<1 \times 10^{-16}$; B: logit(VE_hosp.) = 1.74+0.708 $\log _{2}$ (Neut $A b) ; P<1 \times 10^{-16}$; C: logit(VE_hosp.) = 1.76+0.542 ${ }^{\overline{ }} \log _{2}($ Neut $A b) ; P<1 \times 10^{-16}$ ). 
182

Protection against symptomatic disease showed even larger declines. VE against symptomatic disease for waned neutralizing antibody titers (which were $~ 8$-fold lower than titers in recently vaccinated individuals; (5)) with Pfizer or Moderna against Omicron were far lower than the range of our data and VE was near 0 . For recently vaccinated or boosted vaccinees with Pfizer and Moderna, relative risk increased 4 to 10-fold; e.g. VE was $83.1 \%(95 \% \mathrm{Cl}$ : 82.9-83.2) against Delta for recently vaccinated Pfizer recipients but only 21.4\% (95\% Cl: 17.2-26.4) against Omicron (Fig. 2B). Similar reductions in protection were apparent for documented infections, all infections, and transmission given infection (Table S3).

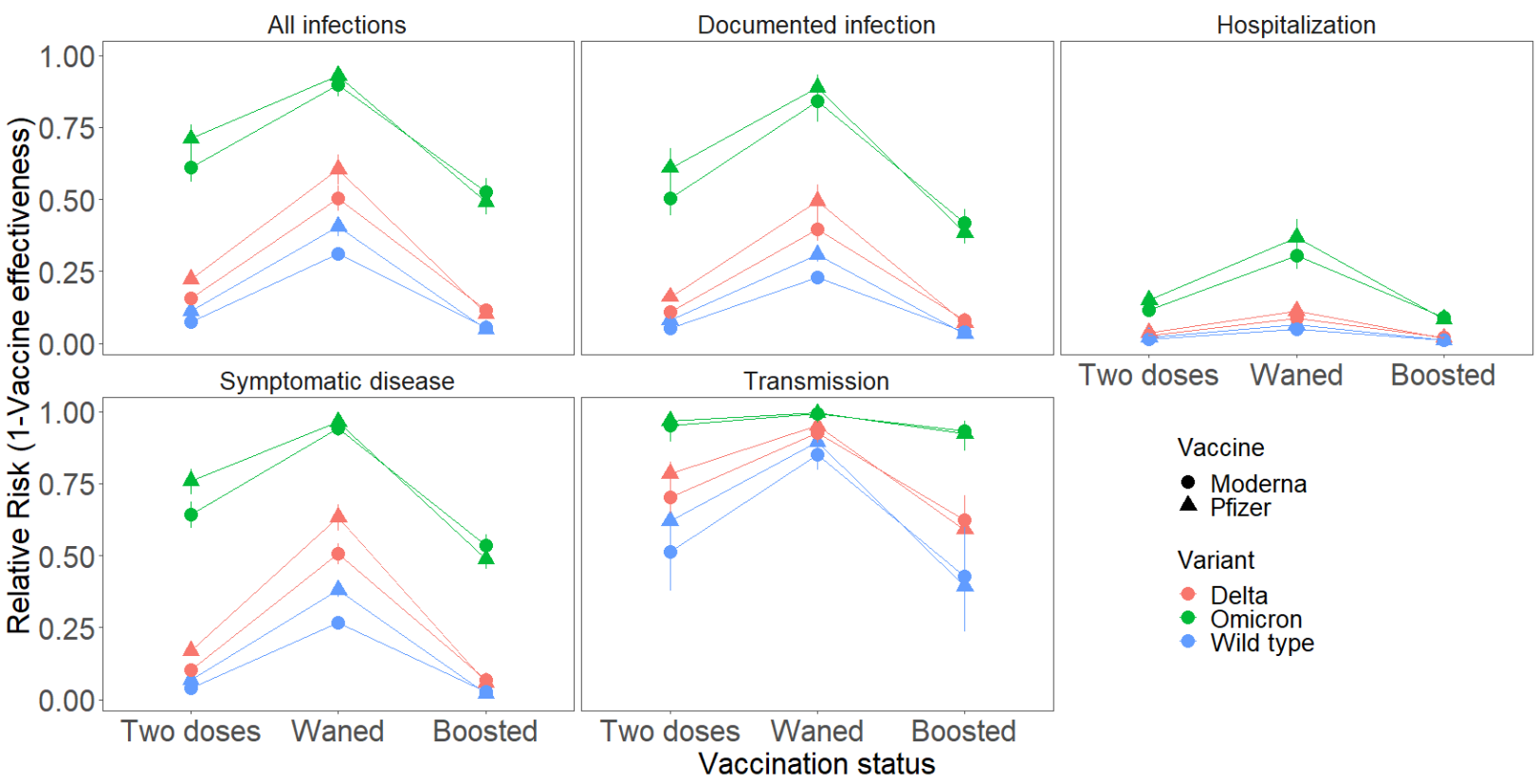

Fig 6. Estimated relative risk (1-VE) for five endpoints, two vaccines, and three variants for three vaccine statuses (shortly after vaccination(two doses), 6 months (Waned), and shorty after a $3^{\text {rd }}$ dose (Boosted).

\section{Discussion}

The emergence and rapid growth of Omicron, a new variant of SARS-CoV-2 with numerous known and novel mutations in the spike protein, has created an urgent need to estimate the impact on protection from vaccines. The fastest way to assess immune escape is by measuring neutralizing antibody titers with sera from vaccinated individuals, but translating these data into estimates of VE across variants had yet to be done. We have shown that ratios of normalized neutralizing antibody titers (normalized to WT virus) among virus variants are moderately repeatable, despite single estimates of normalized neutralizing antibody titers vary over an order of magnitude. We also showed that VE vs hospitalization, symptomatic disease, and documented infection were strongly correlated with neutralizing antibody titer ratios across a 30 to 100 fold range of neutralizing antibody titers, but we had to use variation across both vaccines and variants to span a sizeable range of neutralizing antibody titer. 
We used these relationships to estimate VE for Omicron for vaccinees under a range of states (recently vaccinated, waned and boosted). The 40-fold estimated reduction in neutralizing antibody titers for Omicron increased the relative risk of hospitalization four to five-fold and increased the risk of symptomatic disease seven to ten-fold for mRNA vaccinees. In contrast, neutralizing antibody titers for individuals that were vaccinated with mRNA vaccines $>4$ months ago will have waned $\sim 8$-fold (5) and would now be so low for Omicron ( 320-fold lower than recent vaccinees against WT) putting them outside the range of our data, and indicating very substantial reductions in VE for hospitalization, and nearly zero protection against symptomatic disease, and documented infection. Boosting neutralizing antibody titers with a third dose of mRNA vaccines increased titers substantially, especially compared to waned levels (5), and restored protection back to waned levels against the Delta variant.

These results should be interpreted in the context of several important caveats. First, and foremost, neutralizing antibody titers are a surrogate for VE, and despite strong correlations between VE and neutralizing antibody titers (4-7), direct studies of VE are critical to determine the full extent of immune evasion of new variants. Specifically, our analyses assume that differences in disease severity of variants are correlated with differences in neutralizing antibody titers. Whether this will hold across the 40 -fold reduction in neutralizing antibody titers is highly uncertain. Second, the extent of Omicron immune evasion is so large relative to previous variants ( 40 -fold vs 7 -fold for Beta), and VE estimates for the next most evasive variant, Beta, were so uncertain that we were unable to estimate vaccine-specific models to estimate VE for Omicron. Instead we assumed that differences in neutralizing antibody titers across vaccines and variants are suitable for estimating VE across variants. Third, the 40 -fold reduction that we used for Omicron required extrapolation well outside of available data and prevented us from providing reliable VE estimates for Omicron for vaccinees with waned immunity.

The Omicron variant of SARS-CoV-2 has grown rapidly in many countries. We have shown that the approximately 40 -fold reduction in neutralizing antibody titers greatly erodes vaccine protection and increases the relative risk of infection and symptomatic disease more than four-fold and hospitalization two-fold. As a result, this variant is likely to spread much more quickly than the Delta variant, especially in highly immunized populations. The most promising tool to mitigate this reduction in protection is third doses.

\section{References}

1. Saad-Roy CM, Morris SE, Metcalf CJE, Mina MJ, Baker RE, Farrar J, et al. Epidemiological and evolutionary considerations of SARS-CoV-2 vaccine dosing regimes. Science. 2021;372(6540):363-70. 2. Wagner CE, Saad-Roy CM, Morris SE, Baker RE, Mina MJ, Farrar J, et al. Vaccine nationalism and the dynamics and control of SARS-CoV-2. Science. 2021;373(6562):eabj7364.

3. Sonabend R, Whittles LK, Imai N, Perez-Guzman PN, Knock ES, Rawson T, et al. Nonpharmaceutical interventions, vaccination, and the SARS-CoV-2 delta variant in England: a mathematical modelling study. The Lancet. 2021. 
medRxiv preprint doi: https://doi.org/10.1101/2021.12.10.21267594; this version posted December 12, 2021. The copyright holder for this preprint (which was not certified by peer review) is the author/funder, who has granted medRxiv a license to display the preprint in perpetuity.

It is made available under a CC-BY-NC-ND 4.0 International license .

248 4. Khoury DS, Cromer D, Reynaldi A, Schlub TE, Wheatley AK, Juno JA, et al. Neutralizing antibody 249 levels are highly predictive of immune protection from symptomatic SARS-CoV-2 infection. Nat Med.

250 2021:1-7.

251 5. Gardner BJ, Kilpatrick AM. Third doses of COVID-19 vaccines reduce infection and transmission 252 of SARS-CoV-2 and could prevent future surges in some populations: a modeling study. medRxiv.

253 2021:2021.10.25.21265500.

254 6. Cohen JA, Stuart RM, Rosenfeld K, Lyons H, White M, Kerr CC, et al. Quantifying the role of 255 naturally- and vaccine-derived neutralizing antibodies as a correlate of protection against COVID-19 256 variants. medRxiv. 2021:2021.05.31.21258018.

257 7. van Gils MJ, Lavell AHA, van der Straten K, Appelman B, Bontjer I, Poniman M, et al. Four SARS258 CoV-2 vaccines induce quantitatively different antibody responses against SARS-CoV-2 variants. 259 medRxiv. 2021:2021.09.27.21264163.

260 8. Chen X, Chen Z, Azman AS, Sun R, Lu W, Zheng N, et al. Neutralizing antibodies against severe 261 acute respiratory syndrome coronavirus 2 (SARS-CoV-2) variants induced by natural infection or 262 vaccination: a systematic review and pooled analysis. Clin Infect Dis. 2021.

263 9. Higdon MM, Wahl B, Jones CB, Rosen JG, Truelove SA, Baidya A, et al. A systematic review of 264 COVID-19 vaccine efficacy and effectiveness against SARS-CoV-2 infection and disease. medRxiv.

265 2021:2021.09.17.21263549.

266 10. Cele S, Jackson L, Khan K, Khoury DS, Moyo-Gwete T, Tegally H, et al. SARS-CoV-2 Omicron has 267 extensive but incomplete escape of Pfizer BNT162b2 elicited neutralization and requires ACE2 for 268 infection. medRxiv. 2021:2021.12.08.21267417.

269 11. Sheward DJ, Kim C, Pankow A, Dopico XC, Martin D, Dillner J, et al. Preliminary Report - Early

270 release, subject to modification Quantification of the neutralization resistance of the Omicron Variant of 271 Concern. https://drivegooglecom/file/d/1CuxmNYj5cpluxWXhjjVmuDqntxXwlfXQ/view. 2021.

272 12. Wilhelm A, Widera M, Grikscheit K, Toptan T, Schenk B, Pallas C, et al. Reduced Neutralization of 273 SARS-CoV-2 Omicron Variant by Vaccine Sera and monoclonal antibodies. medRxiv.

$274 \quad$ 2021:2021.12.07.21267432.

275 
medRxiv preprint doi: https://doi.org/10.1101/2021.12.10.21267594; this version posted December 12, 2021. The copyright holder for this preprint (which was not certified by peer review) is the author/funder, who has granted medRxiv a license to display the preprint in perpetuity.

It is made available under a CC-BY-NC-ND 4.0 International license .

277 Supplemental Tables

278 Table S1. Ratio of neutralizing antibody titers relative to wild-type SARS-CoV-2, 279 obtainted from (8).

\begin{tabular}{|l|r|r|r|r|}
\hline Study (8) & Alpha & Beta & Delta & Gamma \\
\hline Anichini et al. & 1.1 & 4.2 & - & 1.2 \\
\hline Cantoni et al. & $1.6,1.5$ & $1.5,3.4$ & - & $3.5,4.4$ \\
\hline Dupont et al. & $2.8,3,2.2,2.1$, & $7.2,5.3,3.5,2.7$ & & $1.1,1.2,1.2,1,1$. \\
\hline Garcia-Beltran & $1.8,2.9$ & $2.7,6.7$ & - & $1.0,1.2$ \\
\hline et al. & 2.1 & $34.5,41.2,42.4$ & - & 6.7 \\
\hline Leier et al. & $2.6,1.5$ & 12.2 & - & $3.4,1.3$ \\
\hline Liu et al. & 0.8 & 2.7 & - & 1.2 \\
\hline Lustig et al. & 1.5 & 8.8 & 4.3 & 2 \\
\hline McCallum et al. & $1.3,1.2,1.9$ & $3.7,2.1,4.4$ & - & $2.1,1.2,3.3$ \\
\hline Su et al. & 1.4 & 9.1 & - & 1.8 \\
\hline Tang et al. & 3.5 & 12.1 & - & 4.8 \\
\hline van Gils et al. & 1.9 & 5.3 & 4.4 & 2.3 \\
\hline Wu et al. a & $1.2,2.8$ & $6.8,7.3,8.4$ & 1.1 & 3.2 \\
\hline Zhang et al. & 2.2 & 4.6 & - & 1.9 \\
\hline
\end{tabular}


Table S2. Vaccine effectiveness estimates obtained from (9).

\begin{tabular}{|c|c|c|c|c|c|c|c|c|c|c|}
\hline Study & Country & Study design & Variant & $\begin{array}{r}\text { History of } \\
\text { COVID }\end{array}$ & Vaccine & Endpoint & VE & $\begin{array}{r}(95 \% \\
\mathrm{Cl})\end{array}$ & $\begin{array}{r}\text { Days } \\
\text { post } \\
\text { final } \\
\text { dose }\end{array}$ & $\begin{array}{r}\text { Effective } \\
\text { sample } \\
\text { size }\end{array}$ \\
\hline Polinkski et al. & USA & Retrospective & Alpha & Excluded & $\mathrm{J} \& \mathrm{~J}$ & Hospitalization & 0.81 & $\begin{array}{l}(0.79- \\
0.84)\end{array}$ & $14+$ & 1000 \\
\hline $\begin{array}{r}\text { Skowronski et } \\
\text { al. }\end{array}$ & Canada & Test-Negative & Alpha & Excluded & Pfizer & Hospitalization & 0.96 & $\begin{array}{l}(0.83- \\
0.99)\end{array}$ & $14+$ & 30 \\
\hline Andrews et al. & England & Test-Negative & Alpha & Excluded & Pfizer & Hospitalization & 0.979 & $\begin{array}{l}(0.914- \\
0.995)\end{array}$ & $14+$ & 40 \\
\hline Andrews et al. & England & Test-Negative & Alpha & Excluded & Astrazeneca & Hospitalization & 0.939 & $\begin{array}{l}(0.849- \\
0.975)\end{array}$ & $14+$ & 62 \\
\hline Stowe & UK & Test-Negative & Alpha & Unknown & Pfizer & Hospitalization & 0.95 & $\begin{array}{l}(0.78- \\
0.99)\end{array}$ & $14+$ & 25 \\
\hline Stowe & UK & Test-Negative & Alpha & Unknown & Astrazeneca & Hospitalization & 0.86 & $\begin{array}{l}(0.53- \\
0.96)\end{array}$ & $14+$ & 10 \\
\hline Haas et al. & Israel & Retrospective & Alpha & Excluded & Pfizer & Hospitalization & 0.972 & $\begin{array}{l}(0.968- \\
0.975)\end{array}$ & $7+$ & 9400 \\
\hline Martinez-Bas & Spain & $\begin{array}{r}\text { Prospective } \\
\text { Cohort }\end{array}$ & Alpha & Excluded & Pfizer & Hospitalization & 0.94 & $\begin{array}{l}(0.6- \\
0.99)\end{array}$ & $14+$ & 7 \\
\hline
\end{tabular}




\begin{tabular}{|c|c|c|c|c|c|c|c|c|c|c|}
\hline Nasreen et al. & Canada & Test-Negative & Alpha & Excluded & Pfizer & $\begin{array}{r}\text { Hospitalization } \\
\text { or Death }\end{array}$ & 0.96 & $\begin{array}{l}(0.92- \\
0.97)\end{array}$ & $14+$ & 700 \\
\hline Nasreen et al. & Canada & Test-Negative & Alpha & Excluded & Moderna & $\begin{array}{r}\text { Hospitalization } \\
\text { or Death }\end{array}$ & 0.95 & $\begin{array}{l}(0.41- \\
0.99)\end{array}$ & $14+$ & 300 \\
\hline Nasreen et al. & Canada & Test-Negative & Alpha & Excluded & Astrazeneca & $\begin{array}{r}\text { Hospitalization } \\
\text { or Death }\end{array}$ & 0.92 & $\begin{array}{l}(0.967- \\
0.972)\end{array}$ & $14+$ & 4 \\
\hline Haas et al. & Israel & $\begin{array}{r}\text { Retrospective } \\
\text { cohort }\end{array}$ & Alpha & Excluded & Pfizer & $\begin{array}{r}\text { Symptomatic } \\
\text { disease }\end{array}$ & 0.97 & $\begin{array}{l}(0.73- \\
0.88)\end{array}$ & $7+$ & 1760 \\
\hline $\begin{array}{r}\text { Martinez-Bas } \\
\text { et al. }\end{array}$ & Spain & $\begin{array}{r}\text { Prospective } \\
\text { cohort }\end{array}$ & Alpha & Excluded & Pfizer & $\begin{array}{r}\text { Symptomatic } \\
\text { disease }\end{array}$ & 0.82 & $\begin{array}{l}(0.86- \\
0.9)\end{array}$ & $14+$ & 109 \\
\hline Nasreen et al. & Canada & Test-Negative & Alpha & Excluded & Pfizer & $\begin{array}{r}\text { Symptomatic } \\
\text { disease }\end{array}$ & 0.88 & $\begin{array}{l}(0.916- \\
0.953)\end{array}$ & $14+$ & 900 \\
\hline $\begin{array}{r}\text { Lopez-Bernal } \\
\text { et al. }\end{array}$ & UK & Test-Negative & Alpha & Excluded & Pfizer & $\begin{array}{r}\text { Symptomatic } \\
\text { disease }\end{array}$ & 0.937 & $\begin{array}{l}(0.938- \\
0.959)\end{array}$ & $14+$ & 693 \\
\hline Andrews et al. & England & Test-Negative & Alpha & Excluded & Pfizer & $\begin{array}{r}\text { Symptomatic } \\
\text { disease }\end{array}$ & 0.95 & $\begin{array}{l}(0.39- \\
0.99)\end{array}$ & $14+$ & 1800 \\
\hline Nasreen et al. & Canada & Test-Negative & Beta & Excluded & Pfizer & $\begin{array}{r}\text { Hospitalization } \\
\text { or Death }\end{array}$ & 0.92 & $\begin{array}{l}(0.86- \\
0.99)\end{array}$ & $14+$ & 4 \\
\hline Nasreen et al. & Canada & Test-Negative & Beta & Excluded & Pfizer & $\begin{array}{r}\text { Symptomatic } \\
\text { disease }\end{array}$ & 0.86 & $\begin{array}{l}(0.75- \\
0.97)\end{array}$ & $14+$ & 1 \\
\hline
\end{tabular}




\begin{tabular}{|c|c|c|c|c|c|c|c|c|c|c|}
\hline Polinkski et al. & USA & Retrospective & Delta & Excluded & J\&J & Hospitalization & 0.85 & $\begin{array}{l}(0.949- \\
0.957)\end{array}$ & $14+$ & 65 \\
\hline Grannis et al. & USA & Test-Negative & Delta & Included & J\&J & Hospitalization & & $\begin{array}{l}(0.9- \\
0.93)\end{array}$ & $14+$ & 11 \\
\hline Grannis et al. & USA & Test-Negative & Delta & Included & Pfizer & Hospitalization & 0.8 & $\begin{array}{l}(0.86- \\
0.92)\end{array}$ & $14+$ & 190 \\
\hline Grannis et al. & USA & Test-Negative & Delta & Included & Moderna & Hospitalization & 0.95 & $\begin{array}{l}(0.82- \\
0.91)\end{array}$ & $14+$ & 300 \\
\hline $\begin{array}{r}\text { Skowronski et } \\
\text { al. }\end{array}$ & Canada & Test-Negative & Delta & Excluded & Pfizer & Hospitalization & 0.98 & $\begin{array}{l}(0.66- \\
0.78)\end{array}$ & $14+$ & 3230 \\
\hline $\begin{array}{r}\text { Skowronski et } \\
\text { al. }\end{array}$ & Canada & Test-Negative & Delta & Excluded & Moderna & Hospitalization & 0.97 & $\begin{array}{l}(0.56- \\
0.73)\end{array}$ & $14+$ & 1200 \\
\hline $\begin{array}{r}\text { Skowronski et } \\
\text { al. }\end{array}$ & Canada & Test-Negative & Delta & Excluded & Astrazeneca & Hospitalization & 0.92 & $\begin{array}{l}(0.909- \\
0.982)\end{array}$ & $14+$ & 160 \\
\hline Andrews et al. & England & Test-Negative & Delta & Excluded & Pfizer & Hospitalization & 0.967 & $\begin{array}{l}(0.734- \\
0.955)\end{array}$ & $14+$ & 8500 \\
\hline Andrews et al. & England & Test-Negative & Delta & Excluded & Astrazeneca & Hospitalization & 0.93 & $\begin{array}{l}(0.71- \\
0.79)\end{array}$ & $14+$ & 8000 \\
\hline Tartof et al. & USA & Retrospective & Delta & Included & Pfizer & Hospitalization & 0.93 & $\begin{array}{l}(0.66- \\
0.77)\end{array}$ & $7+$ & 80 \\
\hline
\end{tabular}




\begin{tabular}{|c|c|c|c|c|c|c|c|c|c|c|}
\hline $\begin{array}{r}\text { Bruxvoort et } \\
\text { al. }\end{array}$ & USA & Test-Negative & Delta & Unknown & Pfizer & Hospitalization & 0.976 & $\begin{array}{l}(0.414- \\
0.672)\end{array}$ & $14+$ & 100 \\
\hline Stowe & UK & Test-Negative & Delta & Unknown & Pfizer & Hospitalization & 0.96 & $\begin{array}{l}(0.78- \\
0.913)\end{array}$ & $14+$ & 40 \\
\hline Stowe & UK & Test-Negative & Delta & Unknown & Astrazeneca & Hospitalization & 0.92 & $\begin{array}{l}(0.75- \\
0.82)\end{array}$ & $14+$ & 25 \\
\hline Nasreen et al. & Canada & Test-Negative & Delta & Excluded & Pfizer & $\begin{array}{r}\text { Hospitalization } \\
\text { or Death }\end{array}$ & 0.98 & $\begin{array}{l}(0.53- \\
0.66)\end{array}$ & $14+$ & 400 \\
\hline Nasreen et al. & Canada & Test-Negative & Delta & Excluded & Moderna & $\begin{array}{r}\text { Hospitalization } \\
\text { or Death }\end{array}$ & 0.98 & $\begin{array}{l}(0.507- \\
0.669)\end{array}$ & $14+$ & 100 \\
\hline Nasreen et al. & Canada & Test-Negative & Delta & Excluded & Astrazeneca & $\begin{array}{r}\text { Hospitalization } \\
\text { or Death }\end{array}$ & 0.9 & $\begin{array}{l}(0.71- \\
0.94)\end{array}$ & $14+$ & 15 \\
\hline Nasreen et al. & Canada & Test-Negative & Delta & Excluded & Pfizer & $\begin{array}{r}\text { Symptomatic } \\
\text { disease }\end{array}$ & 0.92 & $\begin{array}{l}(0.44- \\
0.85)\end{array}$ & $14+$ & 485 \\
\hline $\begin{array}{r}\text { Lopez-Bernal } \\
\text { et al. }\end{array}$ & UK & Test-Negative & Delta & Excluded & Pfizer & $\begin{array}{r}\text { Symptomatic } \\
\text { disease }\end{array}$ & 0.88 & $\begin{array}{l}(0.54- \\
1)\end{array}$ & $14+$ & 715 \\
\hline Tang et al. & Qatar & Test-Negative & Delta & Included & Pfizer & $\begin{array}{r}\text { Symptomatic } \\
\text { disease }\end{array}$ & 0.444 & $\begin{array}{l}(0.41- \\
0.79)\end{array}$ & $14+$ & 205 \\
\hline Andrews et al. & England & Test-Negative & Delta & Excluded & Pfizer & $\begin{array}{r}\text { Symptomatic } \\
\text { disease }\end{array}$ & 0.835 & $\begin{array}{l}(0- \\
0.76)\end{array}$ & $14+$ & 235000 \\
\hline
\end{tabular}




\begin{tabular}{|c|c|c|c|c|c|c|c|c|c|c|}
\hline $\begin{array}{r}\text { Nordstrom et } \\
\text { al. }\end{array}$ & Sweden & $\begin{array}{r}\text { Retrospective } \\
\text { cohort }\end{array}$ & Delta & Excluded & Pfizer & $\begin{array}{r}\text { Symptomatic } \\
\text { disease }\end{array}$ & 0.78 & $\begin{array}{l}(-0.77- \\
0.55)\end{array}$ & $14+$ & 26500 \\
\hline $\begin{array}{r}\text { Skowronski et } \\
\text { al. }\end{array}$ & Canada & Test-Negative & Gamma & Excluded & Pfizer & Hospitalization & 0.95 & $\begin{array}{l}(0.86- \\
0.95)\end{array}$ & $14+$ & 30 \\
\hline Ranzani et al. & Brazil & Test-Negative & Gamma & Excluded & J\&J & Hospitalization & 0.729 & 0.351 & $28+$ & \\
\hline Nasreen et al. & Canada & Test-Negative & Gamma & Excluded & Pfizer & $\begin{array}{r}\text { Hospitalization } \\
\text { or Death }\end{array}$ & 0.94 & 0.59 & $14+$ & 7 \\
\hline Nasreen et al. & Canada & Test-Negative & Gamma & Excluded & Pfizer & $\begin{array}{r}\text { Symptomatic } \\
\text { disease }\end{array}$ & 0.9 & 0.76 & $14+$ & 50 \\
\hline Nasreen & Canada & $\begin{array}{r}\text { Test negative } \\
\text { case-control }\end{array}$ & Alpha & unknown & Moderna & $\begin{array}{r}\text { Symptomatic } \\
\text { disease }\end{array}$ & 0.92 & $\begin{array}{l}(0- \\
0.98)\end{array}$ & $7+$ & 127 \\
\hline Nasreen & Canada & $\begin{array}{r}\text { Test negative } \\
\text { case-control }\end{array}$ & Alpha & unknown & Pfizer & $\begin{array}{r}\text { Symptomatic } \\
\text { disease }\end{array}$ & 0.89 & $\begin{array}{l}(0.73- \\
0.91)\end{array}$ & $7+$ & 600 \\
\hline Lopez Bernal2 & UK & $\begin{array}{r}\text { Test negative } \\
\text { case-control }\end{array}$ & Alpha & excluded & Astrazeneca & $\begin{array}{r}\text { Symptomatic } \\
\text { disease }\end{array}$ & 0.745 & $\begin{array}{l}(0.73- \\
0.85)\end{array}$ & $14+$ & 262 \\
\hline Chemaitelly & Qatar & $\begin{array}{r}\text { Retrospective } \\
\text { cohort }\end{array}$ & Alpha & excluded & Moderna & infection & 1 & $\begin{array}{l}(0.97- \\
0.98)\end{array}$ & $14+$ & 19 \\
\hline Haas & Israel & $\begin{array}{r}\text { Retrospective } \\
\text { cohort }\end{array}$ & Alpha & excluded & Pfizer & infection & 0.953 & $\begin{array}{l}(0.96- \\
0.98)\end{array}$ & $>7$ & 8976 \\
\hline Sheikh & Scotland & $\begin{array}{r}\text { Test negative } \\
\text { case-control }\end{array}$ & Alpha & unknown & Pfizer & infection & 0.92 & $\begin{array}{l}(0.86- \\
0.95)\end{array}$ & $14+$ & 1191 \\
\hline
\end{tabular}




\begin{tabular}{|c|c|c|c|c|c|c|c|c|c|c|}
\hline Abu-Raddad & Qatar & $\begin{array}{r}\text { Test negative } \\
\text { case-control }\end{array}$ & Alpha & unknown & Pfizer & infection & 0.9 & $\begin{array}{l}(0.963- \\
0.97)\end{array}$ & $>14$ & 385 \\
\hline Abu-Raddad & Qatar & $\begin{array}{r}\text { Retrospective } \\
\text { cohort }\end{array}$ & Alpha & unknown & Pfizer & infection & 0.87 & $\begin{array}{l}(0.924- \\
0.935)\end{array}$ & $>14$ & 209 \\
\hline Sheikh & Scotland & $\begin{array}{r}\text { Test negative } \\
\text { case-control }\end{array}$ & Alpha & unknown & Astrazeneca & infection & 0.73 & $\begin{array}{l}(0.84- \\
0.96)\end{array}$ & $14+$ & 223 \\
\hline Martinez-Baz & Spain & $\begin{array}{r}\text { Prospective } \\
\text { cohort }\end{array}$ & Alpha & excluded & Pfizer & infection & 0.65 & $\begin{array}{l}(0.928- \\
0.992)\end{array}$ & $14+$ & 126 \\
\hline Chemaitelly & Qatar & $\begin{array}{r}\text { Test negative } \\
\text { case-control }\end{array}$ & Beta & unknown & Moderna & infection & 0.96 & $\begin{array}{l}(0.853- \\
0.901)\end{array}$ & $14+$ & 122 \\
\hline Chemaitelly & Qatar & $\begin{array}{r}\text { Retrospective } \\
\text { cohort }\end{array}$ & Beta & excluded & Moderna & infection & 0.878 & $\begin{array}{l}(0.37- \\
0.509)\end{array}$ & $14+$ & 41 \\
\hline Abu-Raddad & Qatar & $\begin{array}{r}\text { Test negative } \\
\text { case-control }\end{array}$ & Beta & unknown & Pfizer & infection & 0.75 & $\begin{array}{l}(0.833- \\
0.836)\end{array}$ & $>14$ & 459 \\
\hline Abu-Raddad & Qatar & $\begin{array}{r}\text { Retrospective } \\
\text { cohort }\end{array}$ & Beta & unknown & Pfizer & infection & 0.72 & $\begin{array}{l}(0.78- \\
0.79)\end{array}$ & $>14$ & 261 \\
\hline Tang & Qatar & $\begin{array}{r}\text { Test negative } \\
\text { case-control }\end{array}$ & Delta & included & Moderna & $\begin{array}{r}\text { Symptomatic } \\
\text { disease }\end{array}$ & 0.858 & $\begin{array}{l}(0.55- \\
0.75)\end{array}$ & $14+$ & 39 \\
\hline Lopez Bernal2 & UK & $\begin{array}{r}\text { Test negative } \\
\text { case-control }\end{array}$ & Delta & excluded & Astrazeneca & $\begin{array}{r}\text { Symptomatic } \\
\text { disease }\end{array}$ & 0.67 & $\begin{array}{l}(0.57- \\
0.74)\end{array}$ & $14+$ & 314 \\
\hline
\end{tabular}




\begin{tabular}{|c|c|c|c|c|c|c|c|c|c|c|}
\hline Tang & Qatar & $\begin{array}{r}\text { Test negative } \\
\text { case-control }\end{array}$ & Delta & included & Pfizer & $\begin{array}{r}\text { Symptomatic } \\
\text { disease }\end{array}$ & 0.561 & $\begin{array}{l}(0.65- \\
0.92)\end{array}$ & $14+$ & 55 \\
\hline Tang & Qatar & $\begin{array}{r}\text { Test negative } \\
\text { case-control }\end{array}$ & Delta & included & Moderna & infection & 0.861 & $\begin{array}{l}(0.2- \\
0.85)\end{array}$ & $14+$ & 117 \\
\hline Sheikh & Scotland & $\begin{array}{r}\text { Test negative } \\
\text { case-control }\end{array}$ & Delta & unknown & Pfizer & infection & 0.79 & $\begin{array}{l}(0.89- \\
0.97)\end{array}$ & $14+$ & 520 \\
\hline Sheikh & Scotland & $\begin{array}{r}\text { Test negative } \\
\text { case-control }\end{array}$ & Delta & unknown & Astrazeneca & infection & 0.6 & $\begin{array}{l}(0.83- \\
0.95)\end{array}$ & $14+$ & 233 \\
\hline Tang & Qatar & $\begin{array}{r}\text { Test negative } \\
\text { case-control }\end{array}$ & Delta & included & Pfizer & infection & 0.596 & $\begin{array}{l}(0.9- \\
0.98)\end{array}$ & $14+$ & 148 \\
\hline Heath & UK & Clinical Trial & Alpha & unknown & Novavax & $\begin{array}{r}\text { Symptomatic } \\
\text { disease }\end{array}$ & 0.83 & $\begin{array}{l}(0.31- \\
0.77)\end{array}$ & $7+$ & 40 \\
\hline Emary & UK & Clinical Trial & Alpha & unknown & Astrazeneca & $\begin{array}{r}\text { Symptomatic } \\
\text { disease }\end{array}$ & 0.7 & $\begin{array}{l}(0.92- \\
0.97)\end{array}$ & $14+$ & 24 \\
\hline Thomas & $\begin{array}{l}\text { South } \\
\text { Africa }\end{array}$ & Clinical Trial & Beta & unknown & Pfizer & $\begin{array}{r}\text { Symptomatic } \\
\text { disease }\end{array}$ & & $\begin{array}{l}(0.96- \\
0.99)\end{array}$ & $7+$ & 6 \\
\hline Sadoff & $\begin{array}{l}\text { South } \\
\text { Africa }\end{array}$ & Clinical Trial & Beta & unknown & $\mathrm{J} \& \mathrm{~J}$ & $\begin{array}{r}\text { Symptomatic } \\
\text { disease }\end{array}$ & 0.64 & $\begin{array}{l}(0.93- \\
1)\end{array}$ & $28+$ & 26 \\
\hline Shinde & $\begin{array}{l}\text { South } \\
\text { Africa }\end{array}$ & Clinical Trial & Beta & unknown & Novavax & $\begin{array}{r}\text { Symptomatic } \\
\text { disease }\end{array}$ & 0.51 & $\begin{array}{l}(0.67- \\
0.97)\end{array}$ & $7+$ & 6 \\
\hline
\end{tabular}




\begin{tabular}{|c|c|c|c|c|c|c|c|c|c|c|}
\hline Madhi & $\begin{array}{l}\text { South } \\
\text { Africa }\end{array}$ & Clinical Trial & Beta & unknown & Astrazeneca & $\begin{array}{r}\text { Symptomatic } \\
\text { disease }\end{array}$ & 0.1 & $\begin{array}{l}(0.89- \\
0.94)\end{array}$ & $14+$ & 1 \\
\hline Sadoff & Global & Clinical Trial & D614G & unknown & J\&J & $\begin{array}{r}\text { Symptomatic } \\
\text { disease }\end{array}$ & 0.66 & $\begin{array}{l}(0.83- \\
0.99)\end{array}$ & $28+$ & 91 \\
\hline Voysey & Global & Clinical Trial & D614G & unknown & Astrazeneca & $\begin{array}{r}\text { Symptomatic } \\
\text { disease }\end{array}$ & 0.67 & $\begin{array}{l}(0.351- \\
0.911)\end{array}$ & $14+$ & 123 \\
\hline Tanriover & Turkey & Clinical Trial & D614G & unknown & Sinovac & $\begin{array}{r}\text { Symptomatic } \\
\text { disease }\end{array}$ & 0.84 & $\begin{array}{l}(0.59- \\
0.99)\end{array}$ & $14+$ & 30 \\
\hline Soeriaatmadja & Indonesia & Clinical Trial & D614G & unknown & Sinovac & $\begin{array}{r}\text { Symptomatic } \\
\text { disease }\end{array}$ & 0.65 & $\begin{array}{l}(0.76- \\
0.96)\end{array}$ & $14+$ & 9 \\
\hline Baden & USA & Clinical Trial & D614G & unknown & Moderna & $\begin{array}{r}\text { Symptomatic } \\
\text { disease }\end{array}$ & 0.94 & $\begin{array}{l}(0.86- \\
0.96)\end{array}$ & $14+$ & 134 \\
\hline Novavax & $\begin{array}{r}\text { USA, } \\
\text { Mexico }\end{array}$ & Clinical Trial & D614G & unknown & Novavax & $\begin{array}{r}\text { Symptomatic } \\
\text { disease }\end{array}$ & 0.9 & $\begin{array}{l}(0.86- \\
0.91)\end{array}$ & $7+$ & 103 \\
\hline Polack & Global & Clinical Trial & D614G & unknown & Pfizer & $\begin{array}{r}\text { Symptomatic } \\
\text { disease }\end{array}$ & 0.95 & $\begin{array}{l}(0.684- \\
0.794)\end{array}$ & $7+$ & 200 \\
\hline Logunov & Russia & Clinical Trial & D614G & unknown & Sputnik V & $\begin{array}{r}\text { Symptomatic } \\
\text { disease }\end{array}$ & 0.92 & $\begin{array}{l}(0.825- \\
1)\end{array}$ & $21+$ & 140 \\
\hline
\end{tabular}


medRxiv preprint doi: https://doi.org/10.1101/2021.12.10.21267594; this version posted December 12, 2021. The copyright holder for this preprint (which was not certified by peer review) is the author/funder, who has granted medRxiv a license to display the preprint in perpetuity.

It is made available under a CC-BY-NC-ND 4.0 International license .

Table S3. Predicted VE values for Delta and Omicron variants by vaccine, vaccination status (two-dose, waned, or boosted), and endpoint ("All infections" and "Transmission" are based on analyses from (5); all others are from Figure 5). Rows in italics are substantial extrapolations beyond the range of neutralizing antibody data and should be interpreted with extreme caution.

\begin{tabular}{|c|c|c|c|c|}
\hline Vaccine & Endpoint & Variant & Dose & VE (95\% Cl) \\
\hline Pfizer & All infections & Delta & Waned & $39.6(34.6-44.8)$ \\
\hline Pfizer & All infections & Delta & Two dose & 77.7 \\
\hline Pfizer & All infections & Delta & Boosted & $89.9(89.1-90.6)$ \\
\hline Moderna & All infections & Delta & Waned & $49.6(45.3-54)$ \\
\hline Moderna & All infections & Delta & Two dose & 84.4 \\
\hline Moderna & All infections & Delta & Boosted & $88.5(87.7-89.2)$ \\
\hline Pfizer & All infections & Omicron & Waned & $7.1(4.8-10.5)$ \\
\hline Pfizer & All infections & Omicron & Two dose & 29. $(24-34.6)$ \\
\hline Pfizer & All infections & Omicron & Boosted & $51(46.7-55.2)$ \\
\hline Moderna & All infections & Omicron & Waned & $10.4(7.3-14.5)$ \\
\hline Moderna & All infections & Omicron & Two dose & 38.9 \\
\hline Moderna & All infections & Omicron & Boosted & $47.3(42.8-51.9)$ \\
\hline Pfizer & Documented infection & Delta & Waned & $50.5(44.8-56.2)$ \\
\hline Pfizer & Documented infection & Delta & Two dose & 83.9 \\
\hline Pfizer & Documented infection & Delta & Boosted & $92.8(91.4-94)$ \\
\hline Moderna & Documented infection & Delta & Waned & $60.3(56.1-64.4)$ \\
\hline Moderna & Documented infection & Delta & Two dose & 88.9 \\
\hline
\end{tabular}


medRxiv preprint doi: https://doi.org/10.1101/2021.12.10.21267594; this version posted December 12, 2021. The copyright holder for this preprint (which was not certified by peer review) is the author/funder, who has granted medRxiv a license to display the preprint in perpetuity.

It is made available under a CC-BY-NC-ND 4.0 International license .

\begin{tabular}{|c|c|c|c|c|}
\hline Moderna & Documented infection & Delta & Boosted & $91.8(90.4-93.1)$ \\
\hline Pfizer & Documented infection & Omicron & Waned & $11.2(6.8-17.8)$ \\
\hline Pfizer & Documented infection & Omicron & Two dose & 39.1 \\
\hline Pfizer & Documented infection & Omicron & Boosted & $61.5(57.5-65.4)$ \\
\hline Moderna & Documented infection & Omicron & Waned & $15.7(10.4-23.2)$ \\
\hline Moderna & Documented infection & Omicron & Two dose & $49.8(44-55.6)$ \\
\hline Moderna & Documented infection & Omicron & Boosted & $58.1(53.5-62.6)$ \\
\hline Pfizer & Hospitalization & Delta & Waned & $88.8(87.7-89.8)$ \\
\hline Pfizer & Hospitalization & Delta & Two dose & $96.3(96.1-96.5)$ \\
\hline Pfizer & Hospitalization & Delta & Boosted & $98.1(97.8-98.3)$ \\
\hline Moderna & Hospitalization & Delta & Waned & $91.4(90.7-92)$ \\
\hline Moderna & Hospitalization & Delta & Two dose & $97.3(97.1-97.5)$ \\
\hline Moderna & Hospitalization & Delta & Boosted & $97.9(97.6-98.1)$ \\
\hline Pfizer & Hospitalization & Omicron & Waned & $63.1(56.9-68.9)$ \\
\hline Pfizer & Hospitalization & Omicron & Two dose & $85(83.1-86.6)$ \\
\hline Pfizer & Hospitalization & Omicron & Boosted & 91.7 (91.1-92.3) \\
\hline Moderna & Hospitalization & Omicron & Waned & $69.6(64.6-74.2)$ \\
\hline Moderna & Hospitalization & Omicron & Two dose & $88.6(87.5-89.6)$ \\
\hline Moderna & Hospitalization & Omicron & Boosted & $90.9(90.1-91.5)$ \\
\hline Pfizer & Symptomatic disease & Delta & Waned & $36.8(32.4-41.5)$ \\
\hline
\end{tabular}


medRxiv preprint doi: https://doi.org/10.1101/2021.12.10.21267594; this version posted December 12, 2021. The copyright holder for this preprint (which was not certified by peer review) is the author/funder, who has granted medRxiv a license to display the preprint in perpetuity.

It is made available under a CC-BY-NC-ND 4.0 International license .

\begin{tabular}{|c|c|c|c|c|}
\hline Pfizer & Symptomatic disease & Delta & Two dose & $83.1(82.9-83.2)$ \\
\hline Pfizer & Symptomatic disease & Delta & Boosted & $94.2(93.6-94.7)$ \\
\hline Moderna & Symptomatic disease & Delta & Waned & $49.5(45.8-53.2)$ \\
\hline Moderna & Symptomatic disease & Delta & Two dose & $89.6(89.1-90.1)$ \\
\hline Moderna & Symptomatic disease & Delta & Boosted & $93.1(92.5-93.6)$ \\
\hline Pfizer & Symptomatic disease & Omicron & Waned & $3.6(2.4-5.6)$ \\
\hline Pfizer & Symptomatic disease & Omicron & Two dose & $24.1(19.9-29)$. \\
\hline Pfizer & Symptomatic disease & Omicron & Boosted & $51.2(47.7-54.7)$ \\
\hline Moderna & Symptomatic disease & Omicron & Waned & $6(4.1-8.6)$ \\
\hline Moderna & Symptomatic disease & Omicron & Two dose & $35.9(31.5-40.6)$ \\
\hline Moderna & Symptomatic disease & Omicron & Boosted & $46.6(42.7-50.5)$ \\
\hline Pfizer & Transmission & Delta & Waned & $5(2.2-10.9)$ \\
\hline Pfizer & Transmission & Delta & Two dose & 21.6 \\
\hline Pfizer & Transmission & Delta & Boosted & $41(31.1-51.6)$ \\
\hline Moderna & Transmission & Delta & Waned & 7.3 (3.9-13.3) \\
\hline Moderna & Transmission & Delta & Two dose & $30(24.3-36.3)$ \\
\hline Moderna & Transmission & Delta & Boosted & $37.6(29.1-46.8)$ \\
\hline Pfizer & Transmission & Omicron & Waned & $0.6(0.1-3.5)$ \\
\hline Pfizer & Transmission & Omicron & Two dose & $3.2(1.2-8.5)$ \\
\hline Pfizer & Transmission & Omicron & Boosted & $7.7(4.2-13.7)$ \\
\hline Moderna & Transmission & Omicron & Waned & $0.9(0.2-4.4)$ \\
\hline
\end{tabular}


medRxiv preprint doi: https://doi.org/10.1101/2021.12.10.21267594; this version posted December 12, 2021. The copyright holder for this preprint (which was not certified by peer review) is the author/funder, who has granted medRxiv a license to display the preprint in perpetuity.

It is made available under a CC-BY-NC-ND 4.0 International license .

\begin{tabular}{|l|l|l|l|}
\hline Moderna Transmission & Omicron Two dose $4.9(2.1-10.7)$ \\
\hline Moderna Transmission & Omicron Boosted & $6.7(3.4-12.7)$ \\
\hline
\end{tabular}

